This is a self-archived version of an original article. This version may differ from the original in pagination and typographic details.

Author(s): $\begin{aligned} & \text { Basnayaka, Chathuranga M. Wijerathna; Jayakody, Dushantha Nalin, K.; Chang, } \\ & \text { Zheng }\end{aligned}$

Title: Age of Information Based URLLC-enabled UAV Wireless Communications System

Year: 2022

Version: Accepted version (Final draft)

Copyright: @ I IEEE, 2021

Rights: In Copyright

Rights url: http://rightsstatements.org/page//nC/1.0/?language=en

Please cite the original version:

Basnayaka, C. M. W., Jayakody, D. N., \& Chang, Z. (2022). Age of Information Based URLLCenabled UAV Wireless Communications System. IEEE Internet of Things Journal, 9(12), 1021210223. https://doi.org/10.1109/JIOT.2021.3123431 


\title{
Age of Information Based URLLC-enabled UAV Wireless Communications System
}

\author{
Chathuranga M. Wijerathna Basnayaka, Dushantha Nalin K. Jayakody, Senior Member, IEEE and Zheng \\ Chang, Senior Member, IEEE
}

\begin{abstract}
This paper considers an unmanned aerial vehicles (UAV) communication network, where UAV operate as an aerial mobile relay between a source and a destination of the network. To capture the "timeliness" of the received information at the destination node, a new performance metric named age of information (AoI) is considered. In addition, a short packet communication scheme maintains low latency in the proposed UAV wireless communication system. The finite block-length theory investigates the performances of short packet communications scheme in the UAV-assisted wireless communications system. In this paper, the Average Age of Information (AAoI) is estimated by applying the Stochastic Hybrid Systems (SHS) model. The SHS model comprises discrete states represents events that reset the AoI process and continuously dynamics that represent linearly growing age processes. Finally, a closed-form expression for the AAoI of the proposed UAV wireless communication system is derived and it can be used to estimate the optimal altitude, block length and other parameters.
\end{abstract}

Index Terms-Age of information (AoI), finite block length regime, UAV communication, ultra-reliable and low-latency communications (URLLC), stochastic hybrid systems (SHS).

\section{INTRODUCTION}

\section{A. Motivations}

$\mathbf{I}$ $\mathrm{N}$ recent years, the importance of the UAV-assisted communication network has grown as a part of mobile networks such as beyond fifth-generation (5G). In addition, operating a UAV as a mobile relay increases the the reliability and freshness of the information satisfying requirements of future wireless communication and for the advancements of internet-of-things (IoT) applications [1], [2], [3], [4]. It is essential to maintain the timeliness of status updates at the receiver in future IoT networks since most IoT applications are mission-critical where outdated status could lead to collateral damage. Hence, there are considerable research attempts in the wireless communication research domain that are directed towards developing new metrics to measure and capture the

C. M. W. Basnayaka and D. N. K. Jayakody are with the Centre for Telecommunication Research, School of Engineering, Sri Lanka Technological Campus, Padukka 10500, Sri Lanka and School of Computer Science and Robotics, National Research Tomsk Polytechnic University, Tomsk 634050 Russia. D. N. K. Jayakody is also with the Autonoma TechLab, Portugal and Universidade Autónoma de Lisboa, Portugal.(email: chathurangab@sltc.ac.lk, nalin.jayakody@ieee.org).

Zheng Chang is with Department of Mathematical Information Technology, University of Jyväskylä, P.O.Box 35, FIN-40014 Jyväskylä, Finland. (email: zheng.chang@jyu.fi).

This work is funded, in part, by the Academy of Finland Grant No. 343390 and the Framework of Competitiveness Enhancement Program of the National Research Tomsk Polytechnic University, Russia.

Part of this work is presented at the IEEE 93rd Vehicular Technology Conference, 25 April 2021, Finland, [25]. freshness of the received information. As a result, a new performance metric named the age of information (AoI) is proposed to capture the timeliness of received information. Recent research in the literature related to mission-critical realtime IoT applications is now focused on AoI deviating from the traditional timeliness metric, such as latency and delay. [5], [6]. Thus, AoI has received significant attention from the wireless communications community, especially for the design of status update systems, future time-critical applications and mission-critical applications. In addition, there are two approaches to calculate the average AoI, i.e., the traditionally graphical analysis that calculates the area under instantaneous AoI curve and the stochastic hybrid systems (SHS) approach that considers instantaneous AoI as a stochastic process, which has both discrete and continuous behaviors. Compared with the graphical method, the SHS model is more suitable to analyze complex wireless communication systems with transmission errors [7], [8].

5G mobile networks are designed to address three key use cases: enhanced mobile broadband (eMBB), massive machinetype communication (mMTC), and ultra-reliable low latency communication (URLLC). Currently, the application areas of eMBB and mMTC have achieved a satisfactory level [9]. However, achieving the latency and reliability requirements set by the URLLC standard is more challenging than the other two use cases. Traditionally, long code words have been used to maintain reliable wireless communication. On the other hand, short packet communication considers as an essential feature to support low latency [10]. However, short packet communication has a higher transmission error rate than traditional long packet transmission, making it challenging to maintain reliability requirements. As a result, more resources are needed for re-transmissions and redundancy to improve reliability, but they induce high latency. Hence, it is necessary to carefully navigate these two constraints to meet the requirement of URLLC. Thus, it is inappropriate to use conventional asymptotic information-theoretical results deployed for the long packet communication to calculate the block error probability in short packet communication. Hence, finite block-length information theory has been used as a new theoretical tool to analyze short packet communication [11], [12].

It is important to design a new statistical propagation model to predict path loss, shadowing and fading of aerial communication in UAV-enabled communication. It is different from the common propagation model, which are used for terrestrial communication networks. Basically, this is based 
on environment properties [13], [14]. Normally, UAV communications may provide strong Line-of-Sight (LoS) paths between the UAV and transmission nodes in the ground and it strength the received signal level compared to the Non-Lineof-Sight (NLoS) condition. Hence, it is important to calculate LoS probability to develop a proper channel model for the UAV communication system. Besides the channel statistics, it is important to consider how UAV use as an aerial communication node. In the literature, several cooperative communication techniques have been proposed for the UAV-enabled relay communication networks; among them, decode-and-forward (DF) is one of the most commonly used techniques, where the DF technique is deployed in this paper.

\section{B. Related Works}

A large number of studies have recently been conducted to address various issues in UAV-assisted communication networks, where the researchers are more focused on maintaining the freshness of information received by the end-user in a UAV-assisted network. In these studies, mainly, the AoI is employed as the novel performance metric to measure the freshness of the data. The authors in [15] proposed UAV as a mobile relay to reduce the average peak age-of-information for a source-destination pair where they have developed an optimization algorithm to jointly optimize the flight trajectory of the UAV, energy and service time. Moreover, the ageoptimal trajectory planning issue in UAV-enabled wireless sensor networks is studied in [16], in which a UAV is dispatched to gather information from sensor nodes. In [17], the authors investigated the path scheduling of a UAV for data collection from sensor nodes and the AoI metric has been used to measures the freshness of the data gathered. However, completing the data collecting operation while maintaining the freshness of the information is difficult for a single UAV with limited energy storage. Hence, in [18], the authors examined the AoI-optimal data collection problem in multi-UAV-aided wireless sensor networks.

On the other hand, the short packet communication has received significant attention for solving challenging problems with UAV-assisted communication networks [19]. For example, [20] investigated the feasibility of employing UAVs to support URLLC using short packet communication. In addition, this work provides a technique for optimizing both transmit power and block length for a UAV relay system with short packet communications. The authors in [21] evaluated the latency, reliability, and network availability of URLLC-enabled UAV communication systems, as well as optimized the UAV altitude and bandwidth. The authors of the [19] investigated how to combine passive beamforming, resource allocation, and UAV positioning for IoT devices that communicate by UAV and RIS using short data packets with finite block-length constraints. The average packet error probability and effective throughput of the control link in UAV communications are investigated in [22], where the central ground station delivers control signals to the UAV that need URLLC. The authors of [23] proposed a UAV-enabled relay communication system for transmitting latency-critical messages with ultra-high reliability, where the relay operates in amplifier-and-forward mode.
In this work, they have jointly optimized the UAV position and power to reduce the possibility of decoding errors while maintaining latency requirements.

Unlike the previous works, we are interested in investigating the AoI in an URLLC-enabled UAV-assisted communication system. This study aims to analyze information freshness in a UAV-assisted relaying system with the URLLC standard.

\section{Contributions}

As per the best of author's knowledge, this is the first study that investigates the AoI of an URLLC-enabled UAV wireless network. In this paper, a closed-form expression for the AoI in a UAV relay communication network is derived. Furthermore, our study enables us to determine the optimal altitude, blocklength, transmission power and update generation rate that guarantee the freshness of the received information at the destination. Moreover, we have further extended SHS analysis in the [24] for the wireless system with transmission errors. We also extend our previous work on URLLC enabled decode and forward relay [25] by analyzing UAV communication networks where it has different channel statistics compare with terrestrial wireless channels. Due to erroneous transmission in the relay system, it is not easy to use a graphical method in this analysis. Hence, the SHS approach is adopted and the AoI process is modeled as a stochastic hybrid process that considers both continuous and discrete behaviors of the AoI process.

\section{Organization}

The paper is organized as follows. Section II summarizes the AoI and the SHS technique used for the AoI analysis. Section III introduces the URLLC-enabled UAV relay system and a closed-form equation for average age of information (AAoI) is derived. Subsequently, Section IV presents some numerical results and the system performance is analyzed. Finally, we conclude our work in Section V.

\section{BACKGROUNDS}

\section{A. The Age of Information Metric}

Consider a simple communication system containing a source-destination pair as illustrated in Fig.1, in which a source sends fresh updates to a network; then, the network subsequently delivers those updates to a destination. When the generation time of the most recent update received by the destination at timestamp $t$ is $u(t)$, the AoI can be described as a random process as follows:

$$
\Delta(t)=t-u(t)
$$

As illustrated in the Fig.2, it is assumed that at $t=0$ the measurements of AoI starts and the AoI at the destination is set to $\Delta(0)=\Delta_{0}$. The source generate updates at time instant $u_{0}, u_{1}, u_{2}, \ldots$. . These updates pass through a network and the destination receive these updates at time instant $z_{0}, z_{1}, z_{2}, \ldots$, As illustrated in Fig.4, data update $i$ is transmitted from the source at time instant $t=u_{i}$ and it is successfully delivered to 


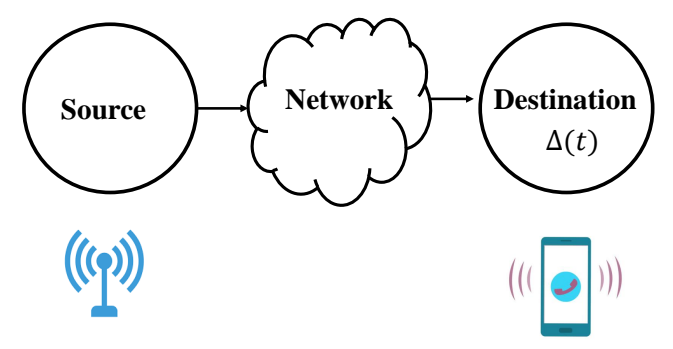

Fig. 1. Simple communication system containing a source-destination pair

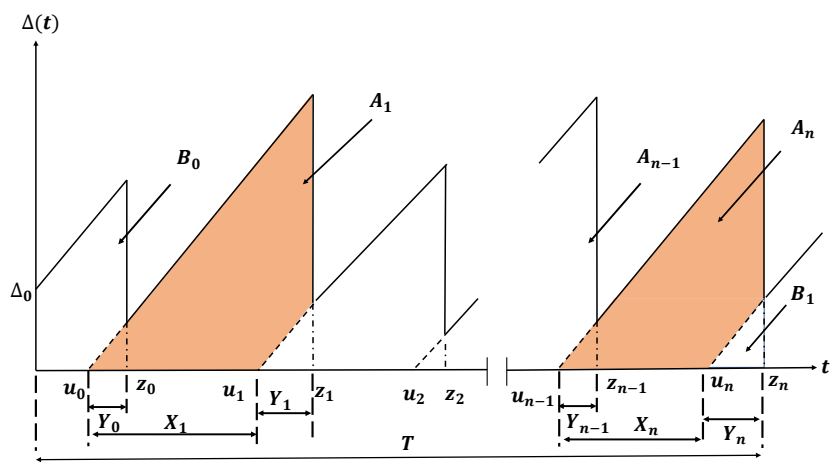

Fig. 2. Evolution of Age of Information $(\Delta(t))$ with the time

the destination at time instant $z_{i}=u_{i}+Y_{i}$. Therefore, the AoI at the destination can be estimated as

$$
\Delta\left(z_{i}\right)=z_{i}-u_{i} .
$$

AoI grows at unit rate until the next update is delivered to the destination. Similarly, AoI just before $i+1$ update is successfully delivered which can be written as,

$$
\Delta\left(z_{i+1}^{-}\right)=Y_{i}+X_{i}
$$

Then, at the time instant $z_{i+1}$, the AoI drops as follows

$$
\Delta\left(z_{i+1}\right)=Y_{i+1} .
$$

Hence, the age process $\Delta(t)$ exhibits the saw-tooth pattern as illustrated in the Fig.2. For a considered time period, time average AoI can be computed using the area under $\Delta(t)$. For this system, the observation time interval considered as $[0, T]$ and $N(T)=\max \left\{n \mid u_{n} \leq T\right\}$ denotes the number of updates by time $T$. The area under the curve can be treated as a sum of the polygon area $B_{0}$, the trapezoidal areas $A_{i}$ for $1 \leq$ $i \leq N(T)\left(A_{1}\right.$ and $A_{n}$ are highlighted in the figure), and the triangular area $B_{1}$ of width $Y_{n}$ over the time interval $\left(u_{n}, z_{n}\right)$ as shown in the Fig.4. Then, the time average age of the proposed system can be estimated by applying graphical methods to saw-tooth age waveform as follows

$$
\Delta_{T}=\frac{1}{T} \int_{0}^{T} \Delta(t) d t=\frac{B_{0}+B_{1}+\sum_{i=1}^{N(T)} A_{i}}{T}
$$

$$
=\frac{B_{0}+B_{1}}{T}+\frac{N(T)}{T} \frac{1}{N(T)} \sum_{i=1}^{N(T)} A_{i}
$$

where

$$
A_{i}=\frac{1}{2}\left(X_{i}+Y_{i+1}\right)^{2}-\frac{1}{2} Y_{i+1}^{2}=Y_{i+1} X_{i}+\frac{1}{2} X_{i}^{2} .
$$

Similar to the work presented in [26], the time average age $\left(\Delta_{T_{n}}\right)$ tends to ensemble average age when $T \rightarrow \infty$, i.e., which can be expressed as

$$
\Delta_{a}=\lim _{T \rightarrow \infty} \Delta_{T} .
$$

For a stationary ergodic updating system in which $X$ is the inter-arrival time between delivered updates and $Y$ is the system time of such a update. When $T \rightarrow \infty, \frac{N(T)}{T} \rightarrow \frac{1}{\mathbb{E}[X]}$, $\frac{1}{N(T)} \sum_{i=1}^{N(T)} A_{i} \rightarrow \mathbb{E}[A]$ and $\frac{B_{0}+B_{1}}{T}$ term goes to zero. Thus, the AAoI can be written as

$$
\text { AAoI }=\Delta_{a}=\frac{\frac{\mathbb{E}\left[X^{2}\right]}{2}+\mathbb{E}[X Y]}{\mathbb{E}[X]} .
$$

In general, $\mathbb{E}[X Y]$ is difficult to estimate since $Y$ and $X$ are dependent random variables for most of the wireless systems. Hence, despite its simplicity, calculating the AoI in complex wireless systems using this type of graphical analysis is challenging. However, the SHS analysis has been introduced as a new tool to analyze AoI replacing traditional graphicalbased AoI analysis [7].

The AoI is widely regarded as a destination-centric metric since it measures the time difference between the current time and the generation time of the most recent update received by the destination. It only takes into consideration packet that are correctly sent to the destination. Moreover, the AoI is strongly affected by the system delay and the update generation rate at the source. In contrast to AoI, the delay is a traditional timeliness metric that measures the latency of an packet and is considered an packet-centric metric. On the other hand, the update (or packet) generation rate at the source does not affect the delay [27].

\section{B. Stochastic Hybrid System for AoI}

In the literature [28], [29], the graphical decomposition approach was the most commonly used technique to estimate AAoI. It has limitations in systems where the packets can be lost or a variety of events need to be accounted e.g., a random access environment. The SHS tool was introduced to address such limitations and it has recently received a considerable attention due to its adaptability and simplicity [24], [30], [31], [32].

Generally, in SHS techniques, the AoI process is modeled as a combination of continuous states $\mathrm{x}(t)$ and discrete states $\mathrm{q}(t)$. Moreover, discrete states $q(t) \in \mathcal{Q}=\{0,1, \cdots, m\}$ capture status of the communication network that can be represented using Markov chain. The evaluation of the age process of the wireless network is represented using continuous vector $\mathrm{x}(t)=\left[x_{0}(t), x_{1}(t), \cdots, x_{n}(t)\right] \in \mathbb{R}^{1 \times n+1}$. Then, SHS model can be described using a graph $(Q, \mathcal{L})$ which contend each discrete state as nodes of the graph and each transaction between discrete states is represented as direct edge. The 
transaction rate from node $q_{l}$ to node $q_{l^{\prime}}$ is $\lambda^{(l)} \delta_{q_{l}, q_{(t)}}$, where Kronecker delta function $\delta_{q_{l}, q_{(t)}}$ limit occurrence of transition only for the state $q_{l}$. For each transition $l$, most of the time, there is a discontinuous jump in the continuous state and it can be represented using linear transition reset mapping as follows:

$$
\mathrm{x}^{\prime}=\mathrm{x} \mathbf{A}_{l}
$$

where $\mathbf{A}_{l} \in\{0,1\}^{(n+1) \times(n+1)}$ is a binary transition reset map matrix. The growth rate of the continuous state at each discrete $q(t)=q$ where $q \in \mathcal{Q}$ given by

$$
\dot{\mathrm{x}}(t)=\frac{\partial \mathrm{x}(t)}{\partial t}=\mathrm{b}_{q}
$$

where $\mathrm{b}_{q}=\left[\mathrm{b}_{q, 0} \mathrm{~b}_{q, 1}, \cdots, \mathrm{b}_{q, n}\right]$ is a vector that contains only binary elements. When $\mathrm{x}_{i}(t)$ grows at a unit rate as a normal age process in the state $q, \mathrm{~b}_{q, j}=1$ and when it is irrelevant to the aging process or does not need to be tracked in the state $q$, $\mathrm{b}_{q, j}=0$. Let $\pi_{\hat{q}}(t)$ denotes the discrete state probabilities for all $\hat{q} \in \mathcal{Q}$ and $\mathrm{v}_{\hat{q}}(t)$ denote the the conditional expectation of the age process, given that $q(t)=\hat{q}$, weighed by the probability of being in $\hat{q}$. Accordingly, we have

$$
\pi_{\hat{q}}(t)=\mathbb{E}\left[\delta_{\hat{q}, q(t)}\right],
$$

and the correlation vector function

$$
\mathrm{v}_{\hat{q}}(t)=\left[v_{\hat{q} 0}(t), \cdots, v_{\hat{q} n}(t)\right],
$$

where

$$
v_{\hat{q} j}(t)=\mathbb{E}\left[x_{j}(t) \delta_{\hat{q}, q(t)}\right], 0 \leq j \leq n .
$$

All transactions $\mathcal{L}$ can be divided into two categories: incoming transitions and outbound transitions. Then, for each state $q$, all incoming transitions labeled as

$$
\mathcal{L}_{q}^{\prime}=\left\{l \in \mathcal{L}: q_{l}^{\prime}=q\right\}
$$

and all outgoing transitions are labeled as

$$
\mathcal{L}_{q}=\left\{l \in \mathcal{L}: q_{l}=q\right\} .
$$

To compute time average age, it is assumed that the Markov chain $q(t)$ is ergotic. Hence, the state probability vector $\pi(t)=\left[\pi_{0}(t), \cdots, \pi_{m}(t)\right]$ always converges to unique stationary vector $\bar{\pi}=\left[\bar{\pi}_{0}, \cdots, \bar{\pi}_{m}\right]$ satisfying

$$
\begin{gathered}
\bar{\pi}_{\bar{q}} \sum_{l \in \mathcal{L}_{\bar{q}}} \lambda^{(l)}=\sum_{l \in \mathcal{L}_{\bar{q}}^{\prime}} \lambda^{(l)} \bar{\pi}_{q_{l}}, \bar{q} \in \mathcal{Q}, \\
\sum_{\bar{q} \in \mathcal{Q}} \bar{\pi}_{\bar{q}}=1
\end{gathered}
$$

As it has been shown in [24], when $\pi(t)=\bar{\pi}, \mathrm{v}(t)=$ $\left[\mathrm{v}_{0}(t), \cdots, \mathrm{v}_{m}(t)\right]$ the system follows first order differential equations for all $\bar{q} \in \mathcal{Q}$ as follows:

$$
\dot{\mathrm{v}}_{\bar{q}}(t)=\mathrm{b}_{\bar{q}} \bar{\pi}_{\bar{q}}+\sum_{l \in \mathcal{L}_{\bar{q}}^{\prime}} \lambda^{(l)} \mathrm{v}_{q_{l}}(t) \mathbf{A}_{l}-\mathrm{v}_{\bar{q}}(t) \sum_{l \in \mathcal{L}_{\bar{q}}} \lambda^{(l)}
$$

Under the ergodicity assumption, the differential equation (18) is stable and each $\mathrm{v}_{\bar{q}}(t)=\mathbb{E}\left[\mathrm{x}(t) \delta_{\bar{q}, q(t)}\right]$ converge to a nonnegative limit $\overline{\mathrm{v}}_{\overline{\mathrm{q}}}$ as $t \rightarrow \infty$. Accordingly, we have

$$
\mathbb{E}[\mathrm{x}]=\lim _{t \rightarrow \infty} \mathbb{E}[\mathrm{x}(t)]=\lim _{t \rightarrow \infty} \mathbb{E}\left[\mathrm{x}(t) \delta_{\bar{q}, q(t)}\right]=\sum_{\bar{q} \in \mathcal{Q}} \overline{\mathrm{v}}_{\overline{\mathrm{q}}},
$$

$$
\overline{\mathrm{v}}_{\bar{q}} \sum_{l \in \mathcal{L}_{\bar{q}}} \lambda^{(l)}=\mathrm{b}_{\bar{q}} \bar{\pi}_{\bar{q}}+\sum_{l \in \mathcal{L}_{\bar{q}}^{\prime}} \lambda^{(l)} \overline{\mathrm{v}}_{q_{l}} \mathbf{A}_{l}, \bar{q} \in \mathcal{Q} .
$$

Here, $x_{0}(t)$ is the age at the destination and AAoI at the destination is calculated as follows [33]:

$$
\Delta=\mathbb{E}\left[x_{0}\right]=\lim _{t \rightarrow \infty} \mathbb{E}\left[x_{0}(t)\right]=\sum_{\bar{q} \in \mathcal{Q}} \bar{v}_{\bar{q} 0}
$$

\section{SySTEM MOdEL}

As shown in Fig. 3, we consider an URLLC-enabled UAV relay network, where the UAV is placed at a altitude of $H$ and it acts as a wireless relay between the source (S) node and the destination (D) node. It is assumed that the altitude of the $\mathrm{S}$ and the $\mathrm{D}$ are negligible as compared to UAV amplitude. The distance between $\mathrm{S}$ and $\mathrm{D}$ is $r$ and it is expect that UAV aerial wireless relay node can freely move between $\mathrm{S}$ and $\mathrm{D}$, while the distance between $\mathrm{S}$ and UAV is $r_{1}=\beta r$ and the distance between UAV relay and D is denoted by $r_{2}=(1-\beta) r$, where $\beta$ denotes the distance dividing factor. The elevation angle of UAV is $\theta$ and if it is measured from the $S, \theta=\theta_{1}=\tan ^{-1}\left(\frac{H}{\beta r}\right)$ and from the $D, \theta=\theta_{2}=\tan ^{-1}\left(\frac{H}{(1-\beta) r}\right)$. In this proposed UAVassisted wireless communication scheme, each transmission time block is divided into two distinct time slots. The source sends data to the UAV relay during the first time span. During the second time slot, the UAV decodes and re-transmits the received data to the destination. It is assumed that no direct communication exists between the source and the destination. The transmitted signal by the source, the received signal at the $\mathrm{UAV}$ realy, the transmitted signal by the UAV, and the received signal at the destination are denoted by $X_{1}, Y_{1}, X_{2}$ and $Y_{2}$, respectively. The received signal at each communication node can be written as

$$
\begin{gathered}
Y_{1}=\sqrt{P_{S}} H_{S, U A V} X_{1}+W_{S, U A V}, \\
Y_{2}=\sqrt{P_{U A V}} H_{U A V, D} X_{2}+W_{U A V, D},
\end{gathered}
$$

where $H_{i j}$ is the channel coefficient of the channel between node $i$ to node $j$ where $i \in\{S, U A V\}$ and $j \in\{U A V, D\}$. The $W_{i j}$ denotes the independent and identically distributed addictive white Gaussian noise (AWGN) of the channel with zero mean and $\sigma^{2}$ variance.

\section{A. Aerial Communication Channel Characterization}

1) Line of sight probability: The possibility that a LoS channel exists between each node is determined by adding the probabilities that each building in the propagation pathway is lower than the height of the ray connecting the two nodes. The height of the ray at the obstruction point can be calculated as

$$
h_{\mathrm{LoS}}=L \tan (\theta) \text {, }
$$

where $L$ is distance from the $\mathrm{S}$ (or $\mathrm{D}$ ) to the obstacle. If structures in the ground are evenly spaced, number of structures between two nodes can be estimated. Assuming area between two nodes has flat or constant slope, the probability that a LoS ray exists is can be calculated as follows:

$$
P_{\mathrm{LoS}}=\prod_{b=1}^{b_{d}} \mathbb{P}\left(h<h_{\mathrm{LoS}}\right)
$$




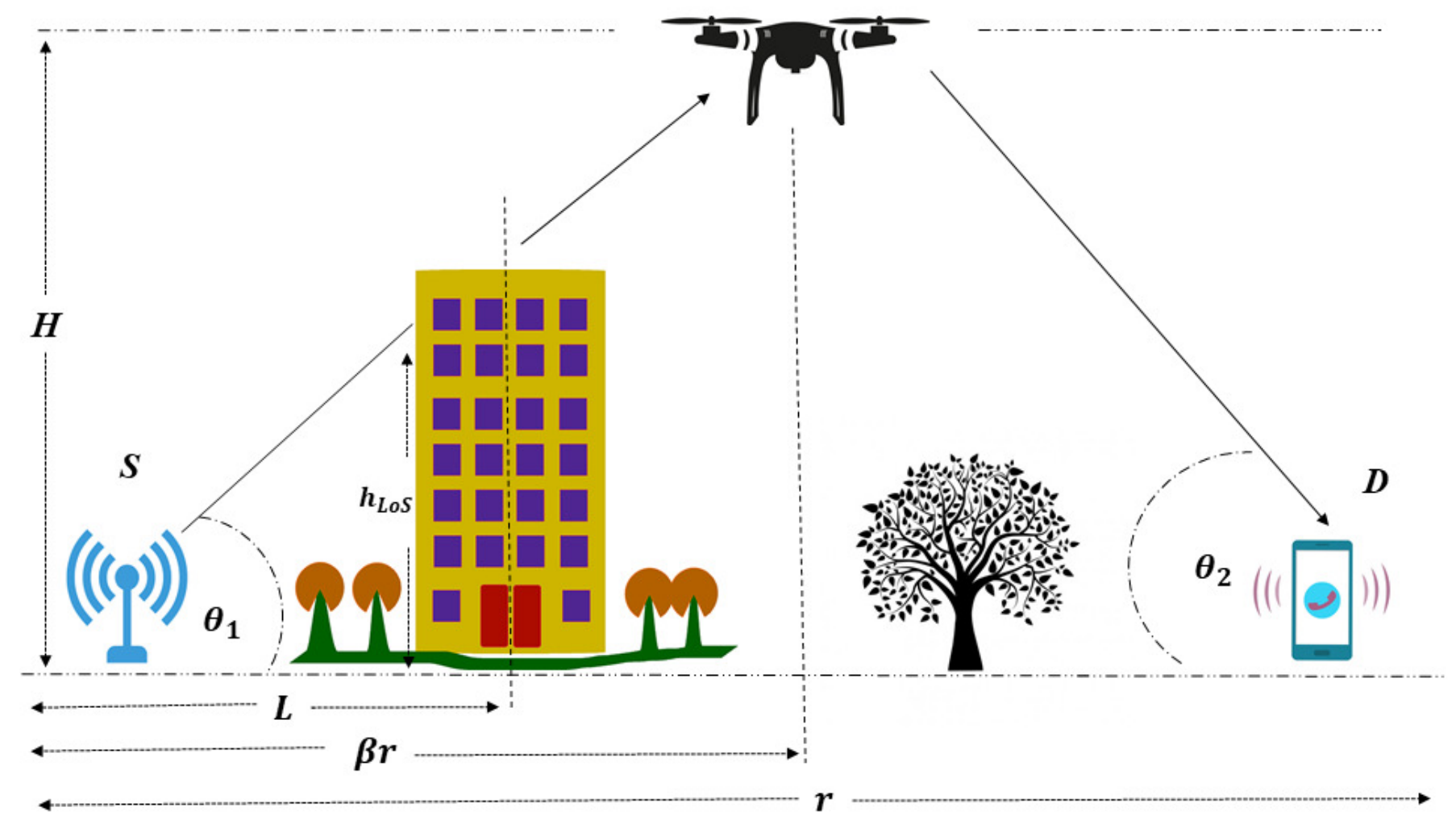

Fig. 3. System model of UAV communication network

where $h$ is the structure height and $b_{d}$ is the number of buildings crossed. The expected number of buildings passed per $\mathrm{km}$ is given by:

$$
b_{1}=\sqrt{Z M},
$$

where $Z$ is the ratio of land area covered by buildings to total land area and $M$ is the average number of buildings per unit area (buildings $/ \mathrm{km}^{2}$ ). Then, the number of buildings crossed by the ray is given by:

$$
b_{r}=\text { floor }\left(r_{j} b_{1}\right),
$$

where $r_{j}(\mathrm{~km}), j \in\{1,2\}$ is the horizontal distance between the transmitter and the receiver and the floor function guarantee that an integer number of terms are contained in (25). The LoS probability can be expressed as closed form expression as follows [34]:

$$
P(\operatorname{LoS})=\prod_{k=0}^{b_{r}-1}\left[1-\exp \left[-\frac{\left(\frac{\left(k+\frac{1}{2}\right) r_{j} \tan \theta}{b_{r}}\right)^{2}}{2 \gamma^{2}}\right],\right.
$$

where $\gamma$ is a scale parameter that describes the buildings' heights distribution that follow Rayleigh probability distri- bution. Akram et al. [35] approximated (28) to a modified Sigmoid function as follows:

$$
P_{\mathrm{LoS}}(\theta)=\frac{1}{1+\rho \exp (-\varphi(\theta-\rho))},
$$

where $\rho$ and $\varphi$ are S-curve parameters that are totally depends on $Z, M$ and $\gamma$ constant. In this paper, this approximation is employed for our analysis due to the complexity of (28).

2) Large-Scale Fading : In the UAV communication, large scale channel statistics such as path loss and shadow fading is effected by the LoS probability. Hence, it is important to derive relationship between LoS probability and large scale channel gain. The additional loss incurred on top of the free space path loss due to the shadowing and scattering caused by buildings and trees is referred to as the excessive path loss, which has a Gaussian distribution. However, instead of focusing on its random behavior, its mean value is considered in this study. Let $\alpha$ be the large scale channel gain of channel between each 
node and it can be obtained as,

$$
\begin{aligned}
-10 \log \left(\alpha_{i j}\right)= & 20 \log (H \csc (\theta))+20 \log \left(\frac{4 \pi f_{c}}{C}\right) \\
& +\eta_{N L O S}+\frac{\eta_{L O S}-\eta_{N L O S}}{1+\rho \exp (-\varphi(\theta-\rho)},
\end{aligned}
$$

where $f_{c}$ and $C$ are the carrier frequency $(\mathrm{Hz})$ and the speed of the light $(\mathrm{m} / \mathrm{s})$, respectively. $\eta_{N L O S}$ and $\eta_{N L O S}$ are the expectation of the additional environment-dependent excessive path loss for the LoS and NLoS components, respectively. In this analysis, it is assumed that all three nodes are stationary when communicating with each other. Hence, the Doppler effect is not taken into account.

3) Small-Scale Fading Statistics: Besides large-scale channel fading characterization, it is essential to investigate the impact of multi-path propagation and small-scale channel characterization of wireless channels. The Rician fading model is often used for UAV communication due to its characteristic nature. The small scale channel gain is denoted as $g_{i j}=\left|H_{i j}^{2}\right|$, where $H_{i j}$ denotes the Rician fading channel coefficient and the distribution of $g_{i j}$ follows a non-central chi-square probability distribution. Then, the probability density function of the small scale channel gain is given by:

$$
f_{g_{i j}}(z)=\frac{(K+1) e^{-K}}{\bar{g}_{i j}} e^{\frac{-(K+1) z}{\bar{s}_{i j}}} I_{0}\left(2 \sqrt{\frac{K(K+1) z}{\bar{g}_{i j}}}\right),
$$

where $z \geq 0, \bar{g}_{i j}=1, I_{0}(\cdot)$ is the zero-order modified Bessel function of the first kind, and $K$ is the Rician factor that depends on the ratio between the power of the LoS component and NLoS components. Then, Rician factor can be expressed as follows [36], [37]:

$$
K=\frac{P_{\mathrm{LoS}}(\theta)}{1-P_{\mathrm{LoS}}(\theta)} .
$$

4) Instantaneous SNR: In this system, transmission power at each transmission node is fixed as $P_{i}$ and the noise power at each receiving node is denoted as $\sigma^{2}$. The instantaneous signal-to-noise ratio (SNR) at the each receiving node is given by:

$$
\gamma_{j}=\frac{\alpha_{i j} g_{i j} P_{i}}{\sigma^{2}}
$$

where $\alpha_{i j}$ and $g_{i j}$ are the large scale channel gain and the small scale channel gain, respectively.

\section{B. Decoding Error Probability under the short packet com- munication}

In this system, we assume that a short packet channel coding scheme is used for encoding. Hence, finite block length information theory was used to evaluate the decoding error probability at the receiving node [38], [39]. The fading coefficients are expected to be constant over the duration of each transmission block in this system, and the receiver has perfect channel state information. Then, the expectation of the decoding error probability at the receiving node can be calculated as follows:

$$
\varepsilon_{j}=\mathbb{E}\left[Q\left(\frac{n_{i, j}\left(\log _{2}\left(1+\gamma_{j}\right)\right)-k}{\left.\sqrt{n_{i, j}\left(\frac{\log _{2}^{2} e}{2}\left(1-\frac{1}{\left(1+\gamma_{j}\right)^{2}}\right)\right.}\right)}\right)\right],
$$

where $\mathbb{E}[$.$] is the expectation operator, Q(x)=\frac{1}{\sqrt{2 \pi}} \int_{x}^{\infty} e^{-\frac{t_{2}}{2}} d t$. It is assume that $k$ number of information bits are contained in a $n_{i, j}$ bits length block. Moreover, under the Rician fading block fading conditions, $\varepsilon_{j}$ can be expressed as

$$
\varepsilon_{j}=\int_{0}^{\infty} f_{\gamma_{j}}(z) Q\left(\frac{n_{i, j}\left(\log _{2}\left(1+\gamma_{j}\right)\right)-k}{\sqrt{n_{i, j}\left(\frac{\log _{2}^{2} e}{2}\left(1-\frac{1}{\left(1+\gamma_{j}\right)^{2}}\right)\right)}}\right) d z,
$$

where $f_{\gamma_{j}}(z)$ denotes the PDF of the received SNR $\left(\gamma_{j}\right)$ at the $j^{\text {th }}$ node and it is given by:

$$
f_{\gamma_{j}}(z)=\frac{(K+1) e^{-K}}{\bar{\gamma}_{j}} e^{\frac{-(K+1) z}{\bar{\gamma}_{j}}} I_{0}\left(2 \sqrt{\frac{K(K+1) z}{\bar{\gamma}_{j}}}\right),
$$

where $\bar{\gamma}_{j}=\frac{P_{i} \alpha_{i j}}{\sigma^{2}}$. Due to the complexity of the Q-function, it is difficult to get a closed-form expression for the overall decoding error probability. Thus, using the approximation technique given in [40] and [12], (35) can be approximated as follows:

$$
\varepsilon_{j} \approx \int_{0}^{\infty} f_{\gamma_{j}}(z) \Theta_{j}(z) d z
$$

where $\Theta_{j}(z)$ denotes the linear approximation of $Q\left(\frac{n_{i, j}\left(\log _{2}\left(1+\gamma_{j}\right)\right)-k}{\left.\sqrt{n_{i, j}\left(\frac{\log _{2}{ }^{2} e}{2}\left(1-\frac{1}{\left(1+\gamma_{j}\right)^{2}}\right)\right.}\right)}\right)$, this can be expressed as [12]

$$
\Theta_{j}\left(\gamma_{j}\right)=\left\{\begin{array}{cc}
1, & \gamma_{j} \leq \phi_{j}, \\
\frac{1}{2}-\beta_{j} \sqrt{n_{i, j}}\left(\gamma_{j}-\psi_{j}\right), & \phi_{j}<\gamma_{j}<\delta_{j}, \\
0, & \gamma_{j} \geq \delta_{j},
\end{array}\right.
$$

where $\beta_{j}=\frac{1}{2 \pi \sqrt{2^{\frac{2 k}{n_{i, j}}}-1}}, \psi_{j}=2^{\frac{k}{n_{i, j}}}-1, \phi_{j}=\psi_{j}-\frac{1}{2 \beta_{j} \sqrt{n_{i, j}}}$ and $\delta_{j}=\psi_{j}+\frac{2 \pi \sqrt{2^{\frac{2 k}{n_{i, j}}}}-1}{2 . \beta_{j} \sqrt{n_{i j}}}$. Using approximation in (38) and [41] the
$\varepsilon_{j}$ is calculated as $\varepsilon_{j}$ is calculated as

$$
\varepsilon_{j} \approx \beta_{j} \sqrt{n_{i, j}} \int_{\phi_{j}}^{\delta_{j}} F_{\gamma_{j}}(z) d z \simeq F_{\gamma_{j}}\left(\psi_{j}\right),
$$

where $F_{\gamma_{j}}$ is the cumulative distribution function (CDF) of the SNR $\gamma_{j}$ and it can be derived as [42] follows:

$$
\begin{aligned}
F_{\gamma_{j}}\left(z, K, \bar{\gamma}_{j}\right) & =\int_{0}^{z} f_{\gamma_{j}}(z) d z \\
& =1-Q_{1}\left(\sqrt{2 K}, \sqrt{\frac{2(K+1) z}{\bar{\gamma}_{j}}}\right),
\end{aligned}
$$


where $Q_{1}(\cdot, \cdot)$ is first order Marcum Q-function. Thus, $\varepsilon_{j}$ can be calculated as

$$
\varepsilon_{j}=1-Q_{1}\left(\sqrt{2 K}, \sqrt{\frac{2(K+1) \psi_{j}}{\bar{\gamma}_{j}}}\right),
$$

However, due to complexity of Marcum Q-function, semilinear approximation is used to get closed form equation for the error probability as follows [43]:

$$
\begin{aligned}
\varepsilon_{j} & \simeq \Xi\left(\sqrt{2 K}, \sqrt{\frac{2(K+1) \psi_{j}}{\bar{\gamma}_{j}}}\right) \\
& \simeq \Xi(\Phi, \Psi),
\end{aligned}
$$

where

$$
\Xi(\Phi, \Psi)=\left\{\begin{array}{c}
0, \text { if } \Psi<c_{1}, \\
\Psi_{0} e^{-\frac{1}{2}\left(\Phi^{2}+\Psi_{0}^{2}\right)} I_{0}\left(\Phi \Psi_{0}\right)\left(\Psi-\Psi_{0}\right)+ \\
1-Q_{1}\left(\Phi, \Psi_{0}\right) \text { if } c_{1} \leq \Psi \leq c_{2}, \\
1, \text { if } \Psi>c_{2},
\end{array}\right.
$$

with

$$
\begin{aligned}
\Psi_{0} & =\frac{\Phi+\sqrt{\Phi^{2}+2}}{2}, \\
c_{1}(\Phi) & =\max \left(0, \Psi_{0}+\frac{Q_{1}\left(\Phi, \Psi_{0}\right)-1}{\Psi_{0} e^{-\frac{1}{2}\left(\Phi^{2}+\Psi_{0}^{2}\right)} I_{0}\left(\Phi \Psi_{0}\right)}\right),
\end{aligned}
$$

and

$$
c_{2}(\Phi)=\Psi_{0}+\frac{Q_{1}\left(\Phi, \Psi_{0}\right)}{\Psi_{0} e^{-\frac{1}{2}\left(\Phi^{2}+\Psi_{0}^{2}\right)} I_{0}\left(\Phi \Psi_{0}\right)} .
$$

When the UAV relay decodes a transmitted block with an error, it may cause transmission failure under the DF relay scheme. Furthermore, even if the UAV correctly decodes the transmitted block, a decoding error at the destination node may cause transmission failure. Then, the overall decoding error probability can be written as [12]

$$
\varepsilon=\varepsilon_{U A V}+\varepsilon_{D}\left(1-\varepsilon_{U A V}\right),
$$

where $\varepsilon_{R}$ is the block error probability at the UAV and $\varepsilon_{D}$ is the block error probability at the destination.

\section{Average Age of Information of the UAV Communication System}

This section estimates the AAoI at the destination (D) of the UAV relay system. The status update packets are generated at the $\mathrm{S}$ and transmitted to the D using UAV relay system. It is considered that mean update generate rate at the $\mathrm{S}$ is $\lambda_{a}$ and UAV relay network transmit update at rate $\mu$. It is assumed that an update in transmission has an error with probability $0 \leq \varepsilon<1$ and it can be calculated using (44). The mean update transmission rate $\mu$ is given by inversely propositional to the mean transmission time per update $\mathbb{E}[T]$ in the UAV relay network and it is given by:

$$
\frac{1}{\mu}=\mathbb{E}[T]=n T_{s}
$$

where $T_{s}$ and $n$ denotes symbol duration and total blocklength, respectively. If the generation time of the freshest update received at time stamp $t$ is $g(t)$, then AoI can be defined as a random process as

$$
x_{0}(t)=t-g(t) .
$$

As illustrated in the Fig.4, it is assumed that at $t=0$ the

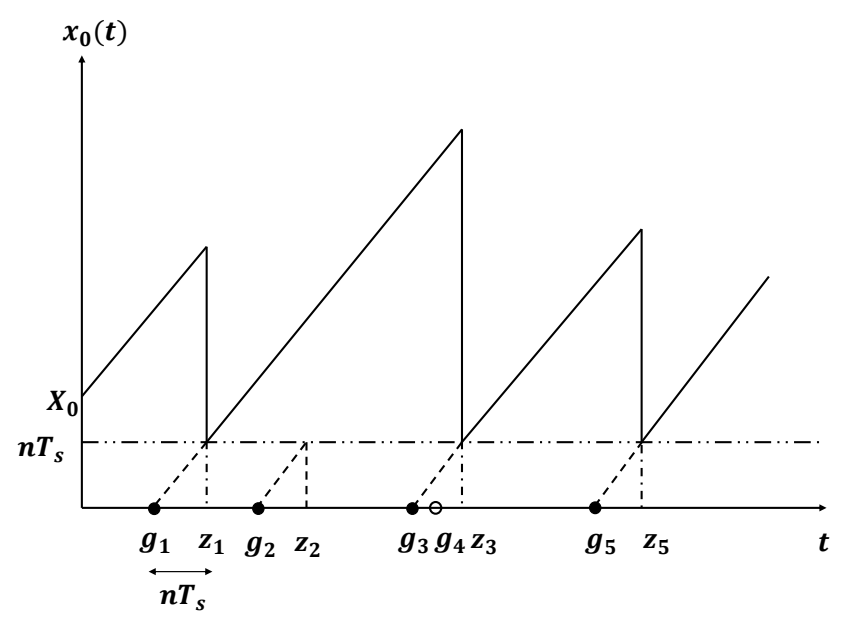

Fig. 4. Evolution of Age of Information $x_{0}(t)$ with the time

measurements of the AoI starts and the AoI at the D is set to $x_{0}(0)=X_{0}$. The $\mathrm{S}$ generate updates at time stamps $g_{1}, g_{2}, \ldots$, and the $\mathrm{D}$ receive these updates at time stamps $z_{1}, z_{2}, \ldots$, if update successfully transmitted by the $\mathrm{S}$ and decoded by the D. As illustrated in Fig.4, data update $i$ is transmitted from the $\mathrm{S}$ at time stamp $t=g_{i}$ and it is successfully delivered to the $\mathrm{D}$ at time stamp $z_{i}=g_{i}+n T_{s}$. Therefore, if update packet delivered successfully, at the time $z_{i}$, the AoI at the D can be estimated as

$$
x\left(z_{i}\right)=n T_{s} .
$$

AoI increases linearly until the next update is successfully delivered to the D. As an example, one packet fails to be decoded at time $z_{2}$, hence, $x_{0}(t)$ continues to increase linearly. Similarly, when there is an update (packet) in transmission, new updates that arrive at the S are blocked and cleared, as an example, update generated at the time $g_{4}$ is not transmitted since at that time the previous update which is generated at the time $g_{3}$ still under the transmission. Hence, the update generated at the time $g_{4}$ does not affect the AoI process. For the considered time period $T_{c}$, time average AoI can be computed using the area under $x_{0}(t)$. Similarly, the time average age of the proposed UAV relay system can be estimated as

$$
\Delta_{T_{c}}=\frac{1}{T_{c}} \int_{0}^{T_{c}} x_{0}(t) d t .
$$

Similar to the work presented in [26], [24], the time average age $\left(\Delta_{T_{c}}\right)$ tends to ensemble average age when $T_{c} \rightarrow \infty$, i.e., which can be expressed as

$$
\Delta=\mathbb{E}\left[x_{0}\right]=\lim _{t \rightarrow \infty} \mathbb{E}\left[x_{0}(t)\right]=\lim _{T_{c} \rightarrow \infty} \Delta_{T_{c}} .
$$

In this paper, SHS techniques are used to evaluate $\mathbb{E}\left[x_{0}\right]$, which is refer to as the AoI at the D. Under the SHS analysis, the AoI process is modeled as combination of continuous 
states $\mathrm{x}(t)$ and discrete states $q(t)$. Where, discrete states capture transmission status of the UAV relay that can be represented using Markov chain and the state space of the Markov chain is $(q(t), \mathrm{x}(t))$, where $q(t) \in \mathcal{Q}=\{0,1\}$, $q=0$ indicates that UAV relay is idle and $q=1$ indicates that UAV relay is on transmission state. The evaluation of the age process at the $\mathrm{D}$ is represented using continuous vector $\mathrm{x}(t)=\left[x_{1}(t), x_{0}(t)\right]$ where $x_{0}(t)$ tracks the age at the $\mathrm{D}$ that sees update that complete transmission without error and $x_{1}(t)$ specifies what $x_{0}(t)$ would become if the packet-in-transmission is delivered. The SHS model for the AoI process at $\mathrm{D}$ the can be illustrated using a graph $(2, \mathcal{L})$ which contents two discrete states (transmission states) of the UAV relay as nodes of the graph and each transaction between transmission states of the UAV relay is represented as direct edge as in the Fig.5. The transaction between the discrete states $q_{l} \rightarrow q_{l}^{\prime}, \forall l \in \mathcal{L}$, and their effect on the continuous state $\mathrm{x}(t)$ are summarized in the Table I using linear mapping of the form $\mathrm{x}^{\prime}=\mathrm{xA}_{l}$. In addition, $\mathrm{v}_{q l} \mathbf{A}_{l}$ included in this table. The transactions presented in the Table I can be explained as follows:

- $l=1:$ An update arrives at the idle system. With the arrival of an update, the $\mathrm{S}$ starts transmission of the update which received and $x_{0}^{\prime}=x_{0}$ is unchanged because the arrival of the update to the source not yield and age reduction at the $\mathrm{D}$ until it is successfully delivered to the destination. However, $x_{1}^{\prime}=0$ since the arriving update is fresh and its age is zero at the instant.

- $l=2$ : An update successfully complete its transmission and is delivered to the D. In this situation, $x_{0}^{\prime}=x_{1}$, corresponding to the age at the $\mathrm{D}$ being reset to the age of the update that just completed transmission. Moreover, $x_{1}^{\prime}=0$ since $x_{1}$ becomes irrelevant when the UAV relay enter to the state 0 .

- $l=3$ : An update complete its transmission with an error. In this situation, $x_{0}^{\prime}=x_{0}$ is unchanged because unsuccessful transmissions not yield and age reduction at the $\mathrm{D}$. Moreover, $x_{1}^{\prime}=0$ since $x_{1}$ becomes irrelevant when UAV relay enter to the state 0 .

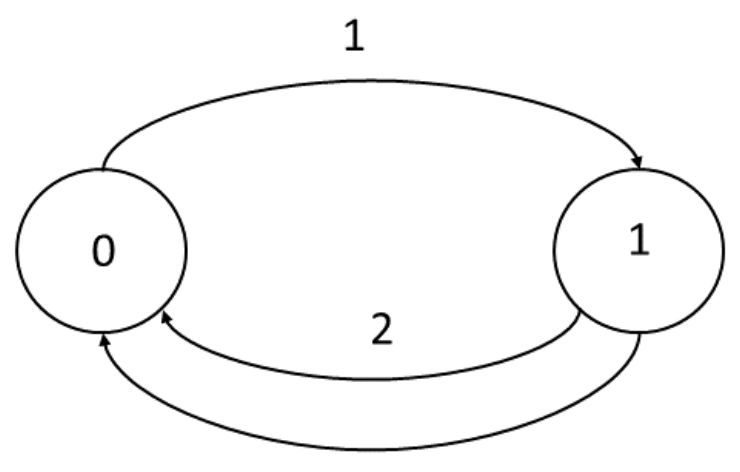

3
TABLE I

TRANSITIONS RATE FOR THE MARKOV CHAIN

\begin{tabular}{|c|c|c|c|c|}
\hline$l$ & $q_{l} \rightarrow q_{l}^{\prime}$ & $\lambda^{(l)}$ & $\mathrm{x} \mathbf{A}_{l}$ & $\mathrm{v}_{q_{l}} \mathbf{A}_{l}$ \\
\hline 1 & $0 \rightarrow 1$ & $\lambda_{a}$ & {$\left[\begin{array}{ll}0 & x_{0}\end{array}\right]$} & {$\left[\begin{array}{ll}0 & v_{00}\end{array}\right]$} \\
\hline 2 & $1 \rightarrow 0$ & $\mu(1-\varepsilon)$ & {$\left[\begin{array}{ll}0 & x_{1}\end{array}\right]$} & {$\left[\begin{array}{ll}0 & v_{11}\end{array}\right]$} \\
\hline 3 & $1 \rightarrow 0$ & $\mu \varepsilon$ & {$\left[\begin{array}{ll}0 & x_{0}\end{array}\right]$} & {$\left[\begin{array}{ll}0 & v_{10}\end{array}\right]$} \\
\hline
\end{tabular}

The evolution of $\mathrm{x}(t)$ at each discrete state $q(t)=q$ is decided by the $\mathrm{b}_{q}$ as follows:

$$
\dot{\mathrm{x}}(t)=\frac{\partial \mathrm{x}(t)}{\partial t}=\mathrm{b}_{q}=\left\{\begin{array}{l}
{\left[\begin{array}{ll}
0 & 1
\end{array}\right] q=0,} \\
{\left[\begin{array}{ll}
1 & 1
\end{array}\right] q=1 .}
\end{array}\right.
$$

To estimate AoI using (21), it is necessary to find $\bar{v}_{\bar{q} 0}$ using (20), in this regards first (16) has been employed to find stationary probabilities and it can be shown that the stationary stationary probability vector satisfies $\bar{\pi} \mathbf{D}=\bar{\pi} \mathbf{Q}$ with

$$
\mathbf{D}=\operatorname{diag}\left[\begin{array}{ll}
\lambda_{a} & \mu
\end{array}\right], \mathbf{Q}=\left[\begin{array}{cc}
0 & \lambda_{a} \\
\mu & 0
\end{array}\right]
$$

Applying (17), the stationary probabilities are given as

$$
\bar{\pi}=\left[\begin{array}{ll}
\bar{\pi}_{0} & \bar{\pi}_{1}
\end{array}\right]=\frac{1}{\lambda_{a}+\mu}\left[\begin{array}{ll}
\mu & \lambda_{a}
\end{array}\right]
$$

Using (20), we can find solution for

$$
\overline{\mathrm{v}}=\left[\begin{array}{ll}
\overline{\mathrm{v}}_{0} & \overline{\mathrm{v}}_{1}
\end{array}\right]=\left[\begin{array}{llll}
\bar{v}_{01} & \bar{v}_{00} & \bar{v}_{11} & \bar{v}_{10}
\end{array}\right] .
$$

Hence, evaluating (17) at $q=0$ and $q=1$ and using Table I, we obtain

$$
\begin{aligned}
& \lambda_{a}\left[\begin{array}{ll}
\bar{v}_{01} & \bar{v}_{00}
\end{array}\right]=\left[\begin{array}{ll}
0 & \bar{\pi}_{0}
\end{array}\right]+\mu(1-\varepsilon)\left[\begin{array}{ll}
0 & \bar{v}_{11}
\end{array}\right]+\mu \varepsilon\left[\begin{array}{ll}
0 & \bar{v}_{10}
\end{array}\right] \\
& \mu\left[\begin{array}{ll}
\bar{v}_{11} & \bar{v}_{10}
\end{array}\right]=\left[\begin{array}{ll}
\bar{\pi}_{1} & \bar{\pi}_{1}
\end{array}\right]+\lambda_{a}\left[\begin{array}{ll}
0 & \bar{v}_{00}
\end{array}\right]
\end{aligned}
$$

By solving (53), (53) and (51) the values of the $\bar{v}_{\bar{q} 0}$ are calculated. Finally, by substituting the result in to (21), the AAoI at the D can be obtained as follows:

$$
\Delta=\frac{1}{(1-\varepsilon) \lambda_{a}}+\frac{1}{(1-\varepsilon) \mu}+\frac{\lambda_{a}}{\mu\left(\lambda_{a}+\mu\right)} .
$$

Approximated AAoI can be calculated applying semi-linearly approximated decoding error probability for (55).

\section{SIMULATION RESULTS}

In this section, numerical results are presented to validate the theoretical derivations. Unless otherwise specified, the simulation parameters are listed in the Table II.

In Fig.6 plots approximated AAoI as a function of the UAV altitude. The approximated AAoI is calculated using (55) with the approximation approach of (42). We can observe from Fig. 6 that the optimal altitude under all environment conditions is $500 \mathrm{~m}$. For low altitude, we have higher AAoI due to the higher error probability caused by the weak LoS. When altitude increase towards its optimal value, AAoI decreases rapidly due to the strong LoS. In this scenario, getting strong LoS channels is become more significant than the losses due 
TABLE II

SIMULATION PARAMETERS [37].

\begin{tabular}{|c|c|}
\hline Parameter & Value \\
\hline Distance between $\mathrm{S}$ and $\mathrm{D}(r)$ & $500 \mathrm{~m}$ \\
\hline Distance dividing factor $(\beta)$ & 0.5 \\
\hline Carrier frequency $\left(f_{c}\right)$ & $6 \mathrm{GHz}$ \\
\hline Speed of the light $(\mathrm{m} / \mathrm{s})$ & $3 \times 10^{8} \mathrm{~ms}^{-1}$ \\
\hline$\eta_{L O S}($ Suburban $)$ & $0.1 \mathrm{~dB}$ \\
\hline$\eta_{N L O S}$ (Suburban) & $21 \mathrm{~dB}$ \\
\hline$\eta_{L O S}($ Urban $)$ & $1 \mathrm{~dB}$ \\
\hline$\eta_{N L O S}($ Urban $)$ & $20 \mathrm{~dB}$ \\
\hline$\eta_{L O S}($ Dense urban $)$ & $1.6 \mathrm{~dB}$ \\
\hline$\eta_{N L O S}$ (Dense Urban) & $23 \mathrm{~dB}$ \\
\hline$\eta_{L O S}$ (High-rise urban) & $2.3 \mathrm{~dB}$ \\
\hline$\eta_{N L O S}$ (High-rise Urban) & $34 \mathrm{~dB}$ \\
\hline Transmission power at source $\left(\mathrm{P}_{S}\right)$ & $5 \mathrm{~mW}$ \\
\hline Transmission power at UAV $\left(\mathrm{P}_{U A V}\right)$ & $5 \mathrm{~mW}$ \\
\hline Symbol duration $\left(T_{S}\right)$ & $17 \mu \mathrm{s}$ \\
\hline$n_{S, U A V}$ & 54 bits \\
\hline$n_{U A V, D}$ & 54 bits \\
\hline$n$ & 108 bits \\
\hline$k$ & 32 bits \\
\hline$\lambda_{a}$ & 529 update $\mathrm{s}^{-1}$ \\
\hline Noise power $\left(\sigma^{2}\right)$ & $-100 \mathrm{dBm}$ \\
\hline
\end{tabular}

to the increased length of the communication link. However, beyond optimal altitude, the path loss dominates the other factors, leading to a higher AAoI. In addition, when altitude between $200 \mathrm{~m}-750 \mathrm{~m}$, approximated AAoI has it minimum for all the other environments except high-rise urban. In this scenario, AAoI goes to its minimum value $4.59 \mathrm{~ms}$ since error probability is approximated as zero. However, compared to the other environmental conditions, high-rise urban environments have low SNR due to the weak LoS; hence, AAoI can not be approximated to its minimum. The suburban environment has the lowest AAoI for all altitudes due to the strong channel condition. In Fig. 7 and Fig. 8 we compare the numerical simulated AAoI with the approximated AAoI for the dense urban environment and the high-rise urban environment, respectively. This approximation is tight for moderate values of $\Psi$ and it is improved for small values of $\Phi$ as in (42). Hence, we can observe that under the dense urban condition, the AAoI approximation is tight for moderate altitude values. Under the high-rise urban condition, simulated AAoI deviates from the approximations for the small or large values of the altitude and the altitudes close to its optimal value. The main reason for this trend is that under these altitudes, the $\Psi$ values are not moderate due to the lower or higher SNR. Fig. 9 shows the AAoI versus the transmission power of the UAV and the source under the different environmental conditions. It is assumed that UAV altitude is $500 \mathrm{~m}$. We can observe that the transmission power significantly affects the AAoI of the proposed UAV communication system. Regardless of

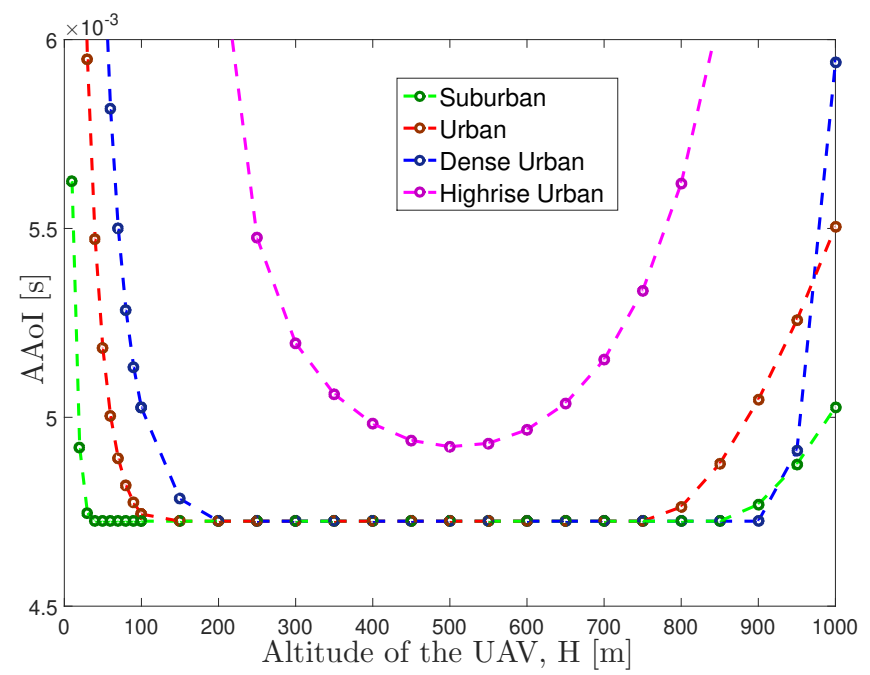

Fig. 6. Approximated AAoI as a function of UAV Altitude

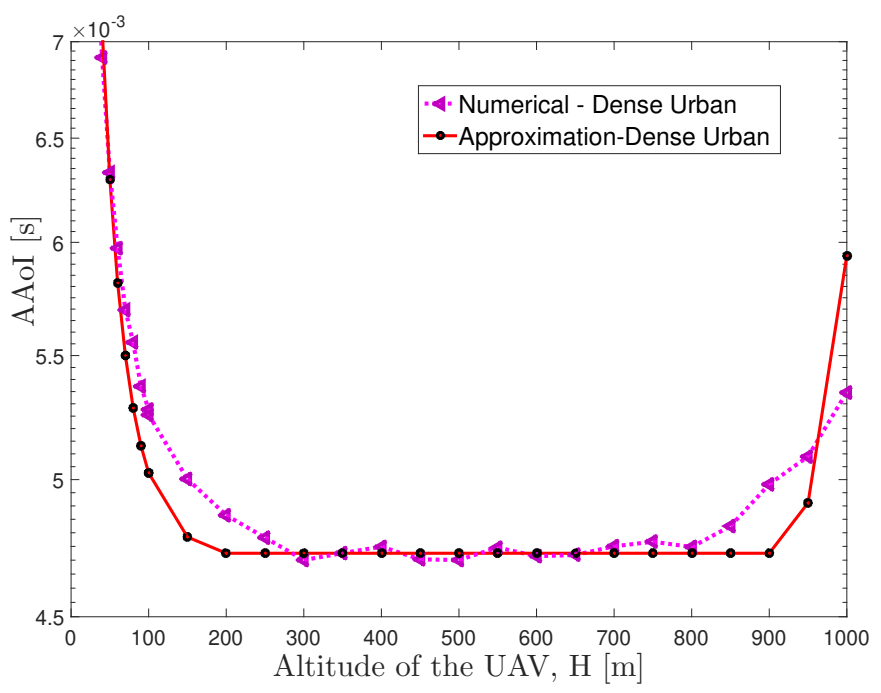

Fig. 7. AAoI as a function of UAV Altitude in the Dense Urban Environment

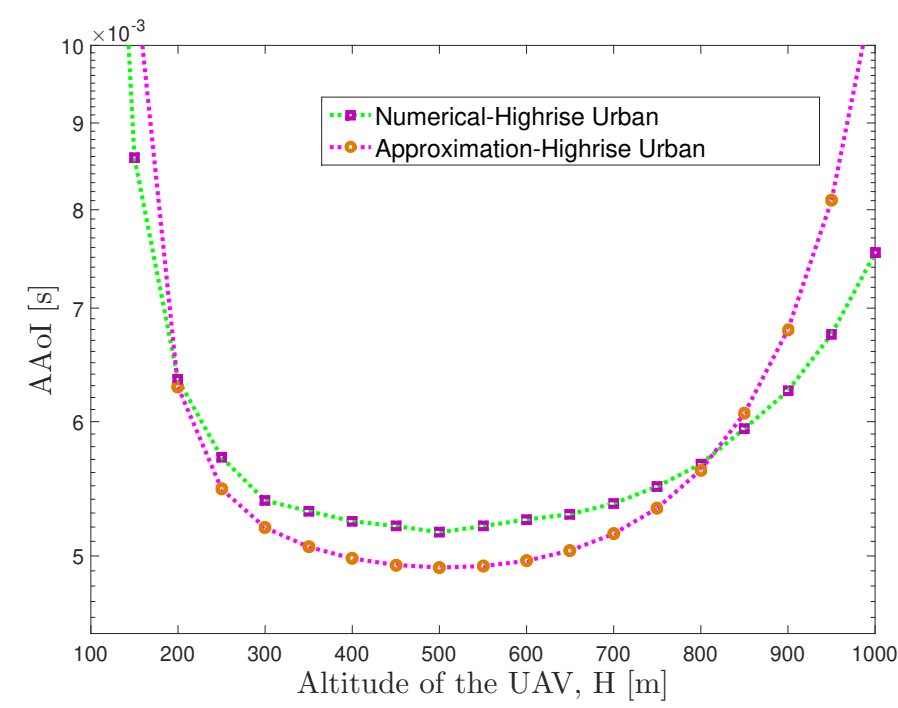

Fig. 8. AAoI as a function of UAV Altitude in the Highrise Urban Environment 
the environmental condition, AAoI is continuously decreasing when the transmission power increases. However, AAoI seems to saturate at $1.5 \mathrm{~mW}$ of transmission power because the number of transmission errors in the wireless network is minimal at that time; hence, the system reaches its lowest AAoI $4.59 \mathrm{~ms}$. However, under high-rise, urban environment conditions, more than $1.5 \mathrm{~mW}$ transmission power is needed to achieve minimum AAoI compared with the other environments. The conclusion of Fig. 9 is that transmission power is a more significant factor that changes the AAoI performance of a UAV-assisted wireless communication system. Fig. 10

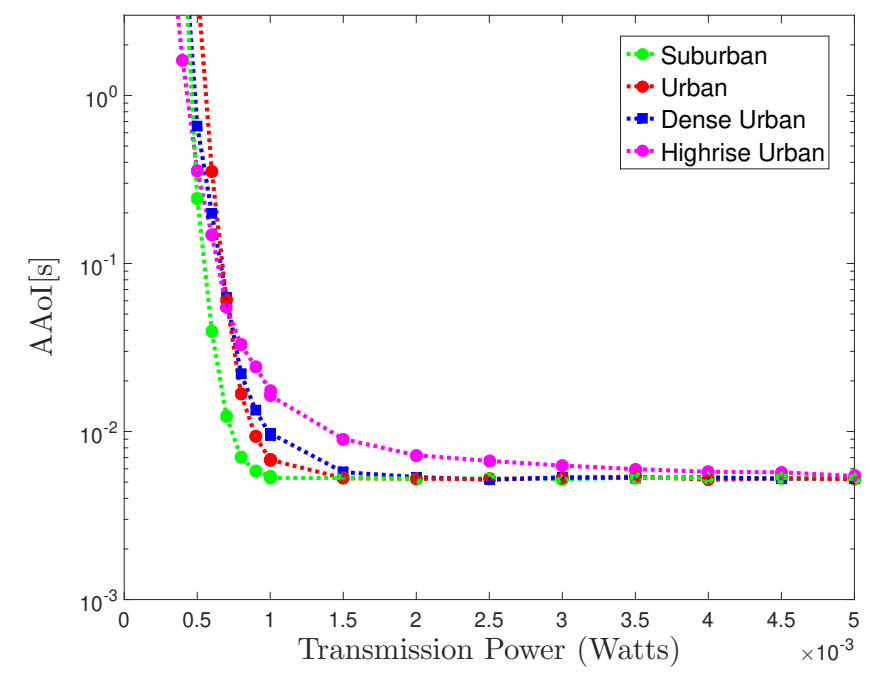

Fig. 9. AAoI as a function of transmission power at UAV and Source

illustrate the effect of the block-length on AAoI when the transmission power is equal to $2 \mathrm{~mW}$. In general, the block length is proportional to the transmission time and increasing block length always increases the system delay of the wireless communication networks. From this figure, we can note that there is an optimal value for the block length and it is observed that for small value of the block-length system has a higher AAoI. Note that small block length values increase the number of transmission errors due to the unsuccessful decoding at the receiving nodes. In this scenario, the effect of the decoding error probability is more significant than the low transmission time on AAoI. On the other hand, increasing block-length from a small value towards optimal value decreases AAoI due to the small number of decoding errors. In this event, the low error probability effect is more significant than increases in the transmission time. However, beyond the optimal value, the effect of the transmission time is more dominant than the decoding error. Thus, from the figure, we can observe that increasing block-length beyond its optimal value rapidly increases the AAoI of the system. The conclusion of Fig. 10 is that maintaining optimal block length is necessary to archive better AAoI.

Fig. 11 plots AAoI as a function of the update generation rate at the source. We see that the AAoI of the proposed UAVassisted communication system is monotonically decreasing with the update generation rate. In this wireless system, no packets are kept in a queue waiting for transmission. Thus,

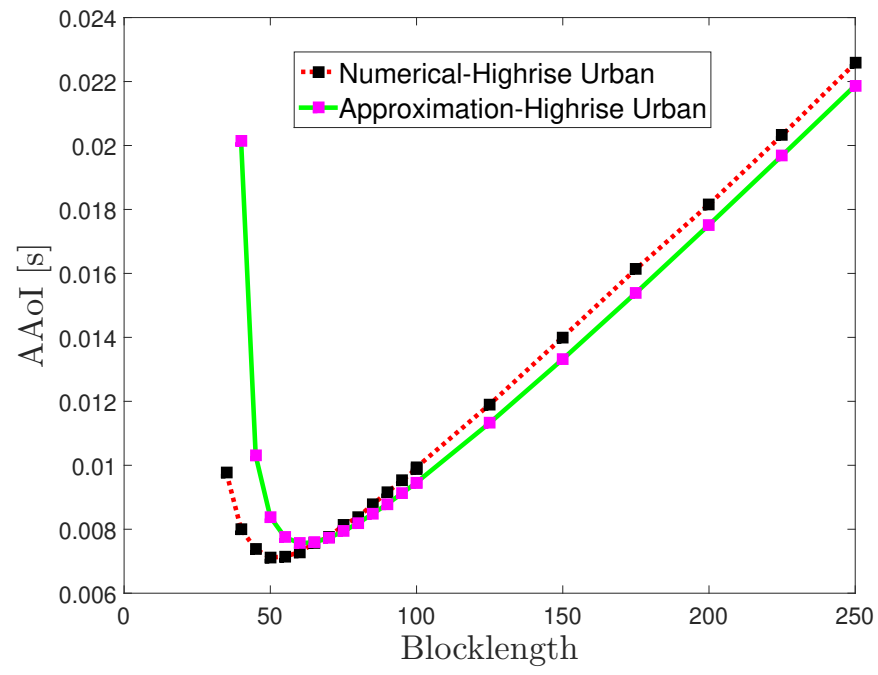

Fig. 10. AAoI as a function of Blocklength in the Highrise Urban Environment

there is no queuing delay in the system and higher update generation rates decrease AAoI. Fig. 12 compares the AAoI

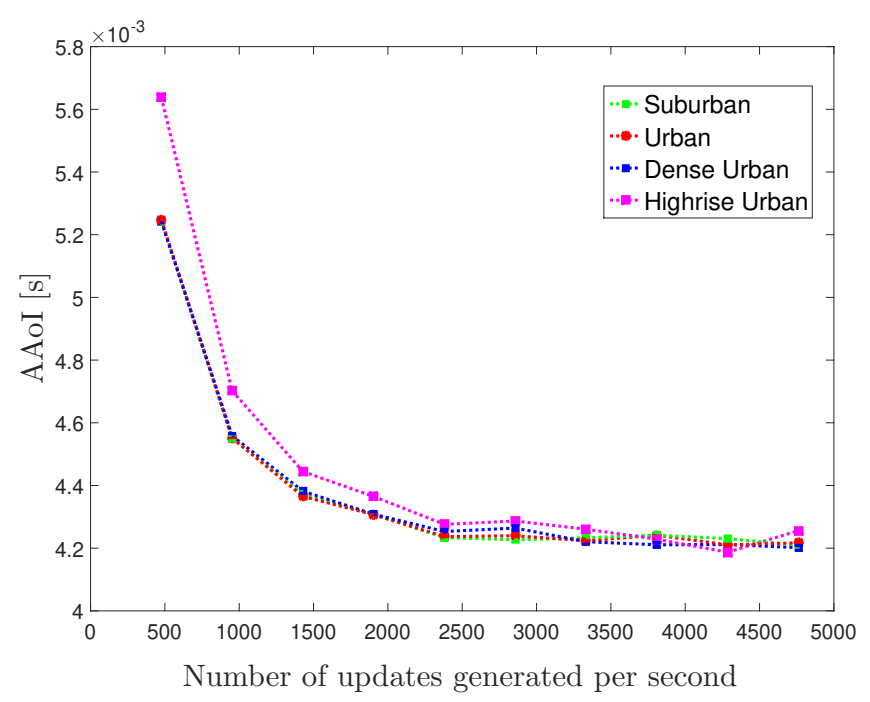

Fig. 11. AAoI as a function of updates generation rate in the Highrise Urban Environment

of the UAV-assisted relay system with that of the direct transmission system. For a fair comparison, we have assumed that the transmission power of the direct transmission system at the $\mathrm{S}$ is $10 \mathrm{~mW}$ and it is equal to the total transmission power of the UAV-assisted relay system. UAV altitude is $500 \mathrm{~m}$. It is observed that by employing a UAV-assisted communication system, we can reduce the AAoI. In addition, the performance gap is more significant for long-distance transmission. Specifically, we highlight the key findings of this simulation results as follows:

- AAoI drops initially and then, subsequently, increases as the UAV altitude increases. When UAV altitude is low, increasing UAV altitude enhances the probability of LoS transmission, which improves the connection reliability. 


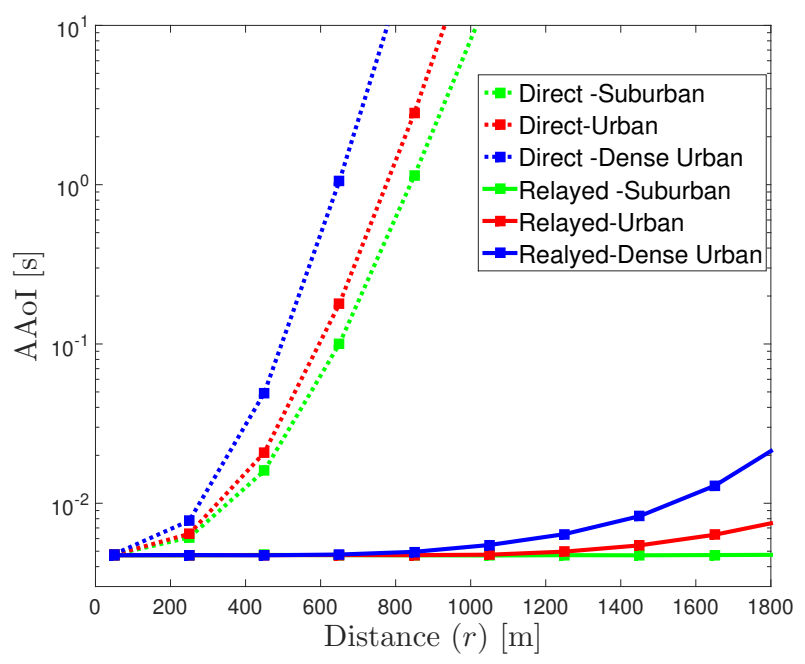

Fig. 12. AAoI as a function of distance between $\mathrm{S}$ and $\mathrm{D}$

As UAV altitude is increased further, a larger path loss is obtained, which dominates the system performance.

- The AAoI of the system decreases monotonically as the update generation rate increases as there is no queuing delay in the system.

- There is an sub-optimal value of block length that minimizes the AAoI. This is mostly due to the trade-off that short block length decreases latency while increasing error probability.

- It has been realized that using a UAV-assisted wireless communication system can reduce the AAoI when compared to with the terrestrial transmission.

\section{CONCLUSIONS}

To determine whether a UAV-assisted relaying system is still significant in terms of information freshness, we estimated the AAoI for an URLLC-enabled UAV-assisted communication system and derived the closed-form expressions for the AAoI. Then, numerical results were provided to determine which UAV-assisted relay or direct transmission systems are best suited for mission-critical IoT applications. Furthermore, AAoI was investigated as a function of block length, UAV altitude, update generation rate, transmission power, and distance. More importantly, numerical results show that UAV-assisted relayed transmission improves the freshness of the information compared to direct transmission.

\section{REFERENCES}

[1] P. S. Bithas, V. Nikolaidis, A. G. Kanatas, and G. K. Karagiannidis, "Uav-to-ground communications: Channel modeling and uav selection," IEEE Trans. Commun., vol. 68, no. 8, pp. 5135-5144, 2020.

[2] L. Sboui, H. Ghazzai, Z. Rezki, and M.-S. Alouini, "Achievable rates of uav-relayed cooperative cognitive radio mimo systems," IEEE Access, vol. 5, pp. 5190-5204, 2017.

[3] A. Sharma, P. Vanjani, N. Paliwal, C. M. W. Basnayaka, D. N. K. Jayakody, H.-C. Wang, and P. Muthuchidambaranathan, "Communication and networking technologies for uavs: A survey," J. Netw. Comput. Appl., p. 102739, 2020.

[4] H. Wang, Q. Yang, Z. Ding, and H. V. Poor, "Secure short-packet communications for mission-critical iot applications," IEEE Trans. Wireless Commun., vol. 18, no. 5, pp. 2565-2578, 2019.
[5] Y. Sun, E. Uysal-Biyikoglu, R. D. Yates, C. E. Koksal, and N. B. Shroff, "Update or wait: How to keep your data fresh," IEEE Trans. Inf. Theory, vol. 63, no. 11, pp. 7492-7508, 2017.

[6] O. T. Yavascan and E. Uysal, "Analysis of slotted aloha with an age threshold," IEEE J. Sel. Areas Commun., vol. 39, no. 5, pp. 1456-1470, 2021.

[7] R. D. Yates, "The age of information in networks: Moments, distributions, and sampling," IEEE Trans. Inf. Theory, vol. 66, no. 9, pp. $5712-5728,2020$.

[8] S. Farazi, A. G. Klein, and D. R. Brown, "Average age of information in multi-source self-preemptive status update systems with packet delivery errors," in Conf. Rec. Asilomar Conf. Signals Syst. Comput. IEEE, 2019, pp. 396-400.

[9] T. N. Weerasinghe, I. A. Balapuwaduge, and F. Y. Li, "Priority-based initial access for urllc traffic in massive iot networks: Schemes and performance analysis," Comput. Netw., vol. 178, p. 107360, 2020.

[10] G. Durisi, T. Koch, J. Östman, Y. Polyanskiy, and W. Yang, "Shortpacket communications over multiple-antenna rayleigh-fading channels," IEEE Trans. Commun., vol. 64, no. 2, pp. 618-629, 2015.

[11] L. Zhang and Y.-C. Liang, "Average throughput analysis and optimization in cooperative iot networks with short packet communication," IEEE Trans. Veh. Technol., vol. 67, no. 12, pp. 11 549-11 562, 2018.

[12] Y. Gu, H. Chen, Y. Li, and B. Vucetic, "Ultra-reliable short-packet communications: Half-duplex or full-duplex relaying?" IEEE Wirel. Commun., vol. 7, no. 3, pp. 348-351, 2017.

[13] A. Al-Hourani, S. Kandeepan, and A. Jamalipour, "Modeling air-toground path loss for low altitude platforms in urban environments," in Proc. IEEE Glob. Commun. Conf. (GLOBECOM). IEEE, 2014, pp. 2898-2904.

[14] H. Hydher, D. N. K. Jayakody, K. T. Hemachandra, and T. Samarasinghe, "Intelligent uav deployment for a disaster-resilient wireless network," Sensors, vol. 20, no. 21, p. 6140, 2020.

[15] M. A. Abd-Elmagid and H. S. Dhillon, "Average peak age-ofinformation minimization in uav-assisted iot networks," IEEE Trans. Veh. Technol., vol. 68, no. 2, pp. 2003-2008, 2018.

[16] J. Liu, X. Wang, B. Bai, and H. Dai, "Age-optimal trajectory planning for uav-assisted data collection," in IEEE Conf. Comput. Commun. Workshops (INFOCOM WKSHPS). IEEE, 2018, pp. 553-558.

[17] G. Ahani, D. Yuan, and Y. Zhao, "Age-optimal uav scheduling for data collection with battery recharging," IEEE Commun. Lett., vol. 25, no. 4, pp. $1254-1258,2020$.

[18] C. Mao, J. Liu, and L. Xie, "Multi-uav aided data collection for age minimization in wireless sensor networks," in Proc. Int. Conf. Wirel. Commun. Signal Process. (WCSP). IEEE, 2020, pp. 80-85.

[19] A. Ranjha and G. Kaddoum, "Urllc facilitated by mobile uav relay and ris: A joint design of passive beamforming, blocklength, and uav positioning," IEEE Internet Things J., vol. 8, no. 6, pp. 4618-4627, 2020.

[20] B. Yu, X. Guan, and Y. Cai, "Joint blocklength and power optimization for half duplex unmanned aerial vehicle relay system with short packet communications," in Proc. Int. Conf. Wirel. Commun. Signal Process. (WCSP). IEEE, 2020, pp. 981-986.

[21] C. She, C. Liu, T. Q. Quek, C. Yang, and Y. Li, "Uav-assisted uplink transmission for ultra-reliable and low-latency communications," in Proc. IEEE Int. Conf. Commun. Workshops (ICC Workshops). IEEE, 2018, pp. 1-6.

[22] K. Wang, C. Pan, H. Ren, W. Xu, L. Zhang, and A. Nallanathan, "Packet error probability and effective throughput for ultra-reliable and lowlatency uav communications," IEEE Trans. Commun., vol. 69, no. 1, pp. 73-84, 2020.

[23] H. Ren, C. Pan, K. Wang, W. Xu, M. Elkashlan, and A. Nallanathan, "Joint transmit power and placement optimization for urllc-enabled uav relay systems," IEEE Trans. Veh. Technol., vol. 69, no. 7, pp. 8003-8007, 2020.

[24] R. D. Yates and S. K. Kaul, "The age of information: Real-time status updating by multiple sources," IEEE Trans. Inf. Theory, vol. 65, no. 3 , pp. 1807-1827, 2018.

[25] C. M. Wijerathna Basnayaka, D. N. K. Jayakody, T. D. Ponnimbaduge Perera, and M. Vidal Ribeiro, "Age of information in an urllcenabled decode-and-forward wireless communication system," in IEEE Veh. Technol. Conf. (VTC2021-Spring), 2021, pp. 1-6.

[26] A. Kosta, N. Pappas, and V. Angelakis, "Age of information: A new concept, metric, and tool," Foundations and Trends in Networking, vol. 12 , no. 3, pp. 162-259, 2017.

[27] R. Talak and E. Modiano, "Age-delay tradeoffs in single server systems," in Proc. IEEE Int. Symp. Inf. Theory (ISIT). IEEE, 2019, pp. 340-344. 
[28] M. Costa, M. Codreanu, and A. Ephremides, "Age of information with packet management," in Proc. IEEE Int. Symp. Inf. Theory (ISIT). IEEE, 2014, pp. 1583-1587.

[29] Y. Inoue, H. Masuyama, T. Takine, and T. Tanaka, "A general formula for the stationary distribution of the age of information and its application to single-server queues," IEEE Trans. Inf. Theory, vol. 65, no. 12, pp. 8305-8324, 2019.

[30] M. Moltafet, M. Leinonen, and M. Codreanu, "Average aoi in multisource systems with source-aware packet management," IEEE Trans. Commun, vol. 69, no. 2, pp. 1121-1133, 2020.

[31] S. K. Kaul and R. D. Yates, "Age of information: Updates with priority," in Proc. IEEE Int. Symp. Inf. Theory (ISIT). IEEE, 2018, pp. 26442648.

[32] A. Maatouk, M. Assaad, and A. Ephremides, "Age-aware stochastic hybrid systems: Stability, solutions, and applications," arXiv preprint arXiv:2109.03919, 2021.

[33] R. D. Yates and S. K. Kaul, "Timely updates by multiple sources: The $\mathrm{m} / \mathrm{m} / 1$ queue revisited," in Proc. Аnпи. Conf. Inf. Sci. Syst. (CISS). IEEE, 2020, pp. 1-6.

[34] P. Data, "Prediction methods required for the design of terrestrial broadband millimetric radio access systems operating in a frequency range of about 20-50 ghz," Draft New Recommendation ITU-R P.[DOC. 3/47], Working Party K, vol. 3, 2003.

[35] A. Al-Hourani, S. Kandeepan, and S. Lardner, "Optimal lap altitude for maximum coverage," IEEE Commun. Lett., vol. 3, no. 6, pp. 569-572, 2014.

[36] M. M. Azari, F. Rosas, K.-C. Chen, and S. Pollin, "Optimal uav positioning for terrestrial-aerial communication in presence of fading," in Proc. IEEE Glob. Commun. Conf. (GLOBECOM). IEEE, 2016, pp. $1-7$.

[37] B. Wang, J. Ouyang, W.-P. Zhu, and M. Lin, "Optimal altitude of uav-bs for minimum boundary outage probability with imperfect channel state information," in Proc. IEEE/CIC Int. Conf. Commun. China (ICCC). IEEE, 2019, pp. 607-611.

[38] Y. Polyanskiy, H. V. Poor, and S. Verdu, "Channel coding rate in the finite blocklength regime," IEEE Trans. Inf. Theory, vol. 56, no. 5, pp. 2307-2359, 2010.

[39] J. Chen, L. Zhang, Y. Liang, X. Kang, and R. Zhang, "Resource allocation for wireless-powered iot networks with short packet communication," IEEE Trans. Wireless Commun., vol. 18, no. 2, pp. 1447-1461, 2019.

[40] B. Makki, T. Svensson, and M. Zorzi, "Finite block-length analysis of the incremental redundancy harq," IEEE Wirel. Commun. Lett., vol. 3, no. 5, pp. 529-532, 2014.

[41] H. Guo, B. Makki, and T. Svensson, "Predictor antennas for moving relays: Finite block-length analysis," in Proc. Int. Conf. Adv. Commun. Technol. Netw. CommNet (CommNet). IEEE, 2020, pp. 1-8.

[42] D. Mishra, S. De, and D. Krishnaswamy, "Dilemma at rf energy harvesting relay: Downlink energy relaying or uplink information transfer?" IEEE Trans. Wireless Commun., vol. 16, no. 8, pp. 4939-4955, 2017.

[43] H. Guo, B. Makki, M.-S. Alouini, and T. Svensson, "A semi-linear approximation of the first-order marcum q-function with application to predictor antenna systems," IEEE open J. Commun. Soc., vol. 2, pp. 273-286, 2021.

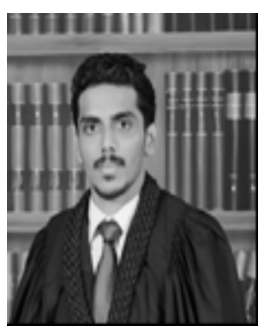

Chathuranga M. Wijerathna Basnayaka was born in Ratnapura, Sri Lanka, in 1993. He received the B.Sc. degree in electrical and information engineering (with honors) from the University of Ruhuna, Sri Lanka. He is currently a doctoral student at National Research Tomsk Polytechnic University in Tomsk, Russia under the supervision of Prof. Dushantha Nalin K. Jayakody and a Research Associate in Sri Lanka Technological Campus (SLTC), Sri Lanka. Prior to joining SLTC in 2019, he worked at University of Sri Jayawardanapura, Sri Lanka and University of Ruhuna, Sri Lanka. Mr.Chathuranga Basnayaka is an Associate Member of the Institution of Engineers, Sri Lanka (IESL).

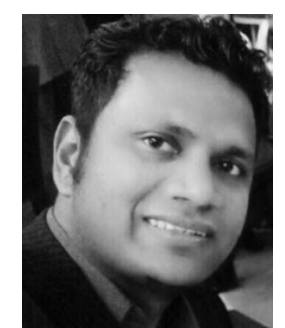

Dushantha Nalin K. Jayakody (S'09-M'14-SM'18) Fellow, IET, received the $\mathrm{Ph}$. D. degree in Electronics and Communications Engineering, from the University College Dublin, Ireland. He received his MSc degree in Electronics and Communications Engineering from the Department of Electrical and Electronics Engineering, Eastern Mediterranean University, Turkey (under the University full graduate scholarship). From 2014 - 2016, he was a Postdoc Research Fellow at University of Tartu, Estonia and University of Bergen, Norway. Since 2021, he is with the Autonoma TechLab, Portugal and Universidade Autónoma de Lisboa, Portugal. He also serves as the Head/ School of Postgraduate Research, Sri Lanka Technological Campus (SLTC), Padukka Sri Lanka and Founding head of Centre of Telecommunication Research, SLTC, Sri Lanka. From 2016 to date, he is a Professor at the School of Computer Science Robotics, National Research Tomsk Polytechnic University (TPU), Russia. He is supervising/supervised $15 \mathrm{PhD}$ students and many master and undergraduate students. He held visiting and/or sabbatical positions at the Centre for Telecommunications Research, University of Sydney, Australia in 2015 and Texas AM University in 2018. Prof. Jayakody has received the best paper award from the IEEE International Conference on Communication, Management and Information Technology (ICCMIT) in 2017 and International Conference on Emerging Technologies of Information and Communications, Bhutan, March 2019. In July 2019, Prof. Jayakody received the Education Leadership Award from the World Academic Congress in 2019. In 2017 and 2018, he received the outstanding faculty award by National Research Tomsk Polytechnic University, Russia. He also received the Distinguished Researcher in Wireless Communications in Chennai, India 2019. Prof. Jayakody has published nearly 200 international peer reviewed journal and conference papers and books. His research interests include PHY and NET layer prospective of $5 \mathrm{G}$ communications technologies such as NOMA for $5 \mathrm{G}$ etc, Cooperative wireless communications, device to device communications, LDPC codes, Unmanned Aerial Vehicle etc. In his career, so far, he has attracted nearly 6M USD research funding. Prof. has organized or co-organized more than 30 workshops, special sessions and IEEE conferences. He currently serves as an Area Editor of the Elsevier Physical Communications Journal, MDPI Information journal, MDPI Sensors and Wiley Internet of Technology Letters. Also, he serves on the Advisory Board of MDPI Multidisciplinary Journal Sci. In addition, he serves as a reviewer for various IEEE Transactions and other journals.

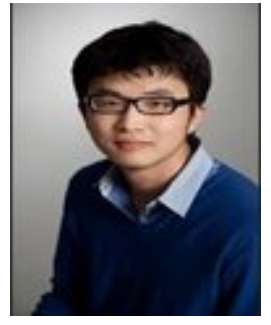

Zheng Chang (Senior Member, IEEE) received the Ph.D. degree from the University of Jyväskylä, Jyväskylä, Finland, in 2013. He has published over 100 papers in journals and conferences, and received best paper awards from IEEE TCGCC and APCC in 2017 and has been awarded as the 2018 IEEE Best Young Researcher for EMEA. He is an Editor of IEEE Wireless Communications Letters, Wireless Networks (Springer), and International Journal of Distributed Sensor Networks. 